\title{
Inhibition of p38 MAPK activity in B-NHL Raji cells by treatment with engineered CD20-specific $T$ cells
}

\author{
LEI JIANG ${ }^{1 *}$, KANG YU ${ }^{2,3}$, JIMEI DU $^{4}$, WUHUA NI ${ }^{1}$, YIXIANG HAN ${ }^{1}$, SHENMENG GAO $^{1}$, \\ HAIYING LI $^{1}$, JIANBO WU ${ }^{1}$, YIHU ZHENG ${ }^{1}$ and YINGXIA TAN ${ }^{1 *}$ \\ ${ }^{1}$ Laboratory of Internal Medicine; ${ }^{2}$ Department of Hematology, The First Affiliated Hospital of Wenzhou Medical College; \\ ${ }^{3}$ Institute of Hematology and Immunology; ${ }^{4}$ Department of Clinical Laboratory Medicine, \\ Wenzhou Medical College, Wenzhou, Zhejiang 325000, P.R. China
}

Received December 16, 2010; Accepted May 12, 2011

DOI: $10.3892 / \mathrm{ol} .2011 .308$

\begin{abstract}
Adoptive immunotherapy with $\mathrm{T}$ cells expressing CD20-specific chimeric T-cell receptors is a promising approach to lymphoma therapy. However, modification of the cellular signaling pathways in target tumor cells by treatment with engineered CD20-specific T cells has yet to be fully elucidated. In this study, the non-Hodgkin's lymphoma Raji cell line was co-cultured with $\mathrm{T}$ cells that were genetically modified with anti-CD20scFvFc/CD28/CD3 $\zeta$ or anti-CD20scFvFc gene. The cytolytic activity of engineered CD20-specific T cells and IL-10 secretion was quantitated by Cytotoxicity and ELISA assays, respectively. The engineered CD20-specific T cells and Raji cells were sorted using flow cytomety for the Western blot analysis. Treatment of Raji cells with T cells genetically modified with anti-CD20scFvFc/CD28/CD3 $\zeta$ chimera (compared to anti-CD20scFvFc) yielded a higher cytotoxicity against Raji cells in vitro. Additionally, we found that engineered CD20-specific $\mathrm{T}$ cells caused a decrease in IL-10 secretion and inhibition of phosphor-STAT3 and Bcl-2 expression in Raji cells, possibly through the down-regulation of p38 MAPK and NF- $\kappa \mathrm{B}$ activity. These results indicate that the treatment of Raji cells with engineered CD20-specific T cells inhibited the cellular p38 MAPK signaling pathways, which enhanced its antitumor activities against CD20-positive tumor cells.
\end{abstract}

\section{Introduction}

Non-Hodgkin's lymphoma (NHL) is one of a large group of cancers of the immune system. Incidence and mortality rates

Correspondence to: Dr Yingxia Tan, Laboratory of Internal Medicine, The First Affiliated Hospital of Wenzhou Medical College, Wenzhou, Zhejiang 325000, P.R. China

E-mail: tanyingxia2005@yahoo.com.cn

${ }^{*}$ Contributed equally

Key words: immunotherapy, CD20, non-Hodgkin's lymphoma, p38 MAPK, interleukin-10 for NHL are currently on the increase worldwide (1). The standard treatment for NHL is chemotherapy. However, relapse eventually occurs and subsequent failure of chemotherapy to control cancer has prompted the development of alternative therapies (2). Mounting evidence shows that adoptive immunotherapy with genetically modified $\mathrm{T}$ cells expressing chimeric T-cell receptors targeting lymphoma-associated antigens is a promising approach for treating this group of diseases. CD20 is exclusively expressed in B-cell lineage and is minimally modulated or shed from the cell surface on more than $95 \%$ of B-cell lymphomas at high copy numbers. CD20 is also minimally internalized on binding antibodies (Ab) (3). These characteristics make CD20 an attractive target for immunotherapy. Engineered T cells expressing a single chain anti-CD20 Ab fused to the T-cell receptor complex CD3 $\zeta$ chain display redirecting MHC-unrestricted CD20-specific lymphoma cell cytolysis (4). The CD3ל chain was previously shown to be sufficient for mediating T-cell activation signals (5). CD3 and CD28 signals were found to be fundamental for cellular proliferation and antigen-induced interleukin-2 (IL-2) secretion of grafted $\mathrm{T}$ cells in an anti-CEA $\mathrm{scFv}$ mediated T-cell adoptive immunotherapy study (6). The two signals can be delivered by one recombinant receptor molecule $(6,7)$. Previously, we reported that engineered CD20-specific $\mathrm{T}$ cells, particularly lysed CD20-positive target tumor cells, secreted IFN- $\gamma$ and IL-2 after binding to their target cells. However, modifications of the cellular signaling pathways in target tumor cells by engineered CD20-specific T cells have yet to be fully elucidated.

In the present study, we observed that treatment of NHL Raji cells with engineered CD20-specific $\mathrm{T}$ cells inhibited p38 MAPK and NF- $\kappa$ B activities and resulted in the inhibition of IL-10 secretion, phosphor (p)-STAT3 and Bcl-2 expression. In addition, the activity of engineered $\mathrm{T}$ cells expressing anti-CD20scFvFc or anti-CD20scFvFc/CD28/ $\mathrm{CD} 3 \zeta$ in vitro was investigated.

\section{Materials and methods}

Cell culture and plasmid DNA. The Burkitt lymphoma cell line, Raji, was cultured in RPMI-1640 with $10 \%$ fetal bovine serum (FBS; Invitrogen, Carlsbad, CA, USA) at $37^{\circ} \mathrm{C}$ in a 
A





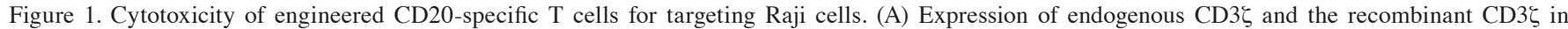
engineered CD20-specific T cells. A 21-kDa band corresponding to wild-type CD3 $\zeta$ was present in the cell lysates of anti-CD20scFvFc and anti$\mathrm{CD} 20 \mathrm{scFvFc} / \mathrm{CD} 28 / \mathrm{CD} 3 \zeta$-transfected PTLs, and a second $\sim 68 \mathrm{kDa}$ band representing the expected anti-CD20scFvFc/CD28/CD3 $\zeta$ protein was detected in cell lysates from anti-CD20scFvFc/CD28/CD3ל-transfected PTLs. (B) Expanded primary T cells (untransfected, anti-CD20scFvFc or anti-CD20scFvFc/ CD28/CD3 $\xi$-transfected PTLS) were incubated with the NHL Raji cells (E/T ratio: 10) for different time points. The lysis of target cells was monitored by a non-radioactive cytotoxicity assay, and data were expressed as the mean $\pm \mathrm{SD}(\mathrm{n}=3)$. ${ }^{\&} \mathrm{P}<0.05$ compared to the untransfected T-cell group; ${ }^{*} \mathrm{P}<0.05$ compared to the anti-CD20scFvFc-transfected T-cell group.

humidified $5 \% \mathrm{CO}_{2}$ incubator. The pLNCX vector containing anti-CD20scFvFc and anti-CD20scFvFc/CD28/CD3 $\zeta$ was constructed as previously described (8).

Generation of genetically modified $T$ cells. Peripheral T-cell lymphomas (PTLs) were isolated from normal donor blood as previously described $(7,8)$. Briefly, peripheral blood mononuclear cells from healthy donors were isolated by Ficoll-Paque (GE Healthcare, Little Chalfont, UK) density gradient centrifugation and cultured in RPMI-1640 containing 10\% FBS, $1 \mu \mathrm{g} / \mathrm{ml}$ PHA-L (Roche, Basel, Switzerland), $30 \mathrm{ng} / \mathrm{ml}$ OKT3 (Wuhan Institute of Biological Products, Wuhan, China) and $50 \mathrm{U} / \mathrm{ml}$ rhIL-2 (Sigma-Aldrich, St. Louis, MO, USA). After 10 days of sustainable culture, cells were analyzed by flow cytometry using a Simultest Imk-Lymphocyte kit (BD, Franklin Lakes, NJ, USA). Plasmid transfection of T cells occurred when $>90 \%$ of the cells were positive for CD3 PTLs $\left(5 \times 10^{7}\right)$ were mixed with $10 \mathrm{mg} / \mathrm{ml}$ salmon sperm DNA (Invitrogen) and $100 \mu \mathrm{g}$ linearized plasmid DNA. Cells were electroporated with Bio-Rad Gene Pulser Xcell at $300 \mathrm{~V}$, $960 \mu \mathrm{A}$ for $2 \mathrm{~min}$. Approximately $48 \mathrm{~h}$ after electroporation, cells were selected by $800 \mu \mathrm{g} / \mathrm{ml} \mathrm{G} 418$ for 7 days and G418resistant PTLs were successfully transfected with recombinant gene for use in this study.

Cytotoxicity assays. The cytolytic activity of engineered CD20-specific $\mathrm{T}$ cells was quantitated using a Cytotoxicity Detection kit (Roche, Indianapolis, IN, USA) according to the manufacturer's instructions and by employing Raji cells as target cells. The target Raji cells were co-cultured with engineered $\mathrm{CD} 20$-specific $\mathrm{T}$ cells ( $\mathrm{E} / \mathrm{T}$ ratio was 10$)$, and incubated at $37^{\circ} \mathrm{C}$ for $0,4,8,12,18,24$ or $48 \mathrm{~h}$. The cytotoxicity assay results were obtained from three independent experiments performed in triplicate and the percentage of specific cytotoxicity was calculated.

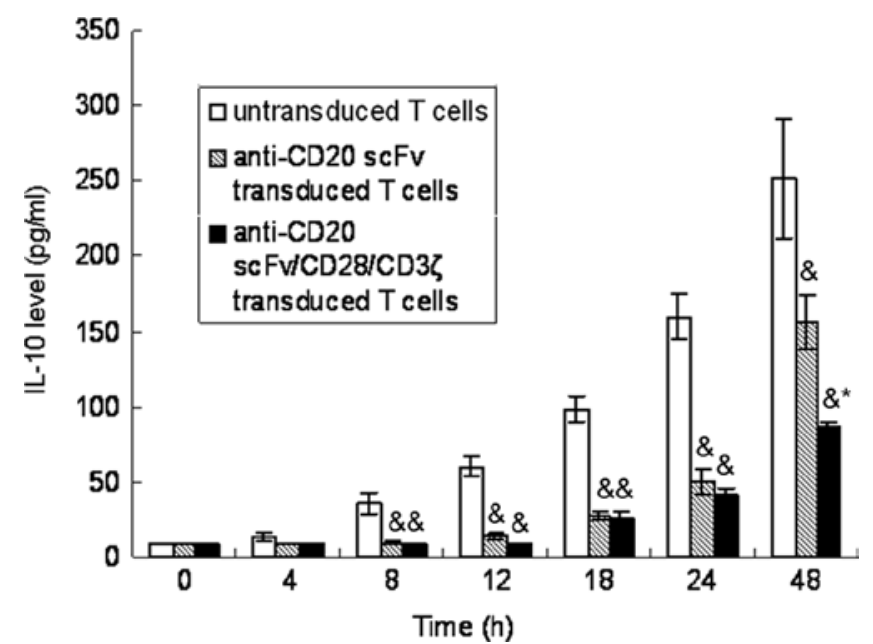

Figure 2. IL-10 production was measured using ELISA. Raji cells were co-cultured with anti-CD20scFvFc, anti-CD20scFvFc/CD28/CD3 transfected or untransfected $\mathrm{T}$ cells for different time points. Data were expressed as the mean $\pm \mathrm{SD}(\mathrm{n}=3)$. ${ }^{\circledR} \mathrm{P}<0.05$ compared to the untransfected T-cell group; " $\mathrm{P}<0.05$ compared to the anti-CD20scFvFc-transfected T-cell group.

IL-10 secretion assay. Engineered CD20-specific T cells were co-cultured with stimulator Raji cells in 24-well assay plates following incubation. Culture supernatants were harvested and used to detect the IL-10 level using Cytokine ELISA assays (R\&D, Minneapolis, MN, USA), according to the manufacturer's instructions.

Western blotting. Raji cells were treated with engineered CD20-specific $\mathrm{T}$ cells and CD3-negative Raji cells were sorted by flow cytometry for the Western blot analysis. Western blots were carried out as previously described $(8,9)$. The primary antibodies used were polyclonal antibodies 
A

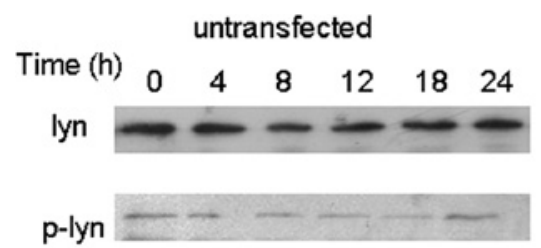

anti-CD20scFvFc
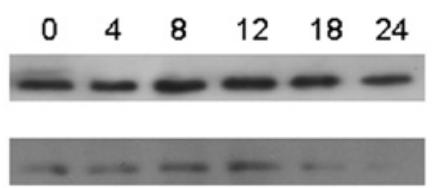

anti-CD20scFvFc/CD28/CD3\}

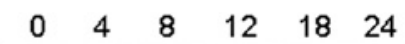

\section{$\mathrm{mon}=$}


B
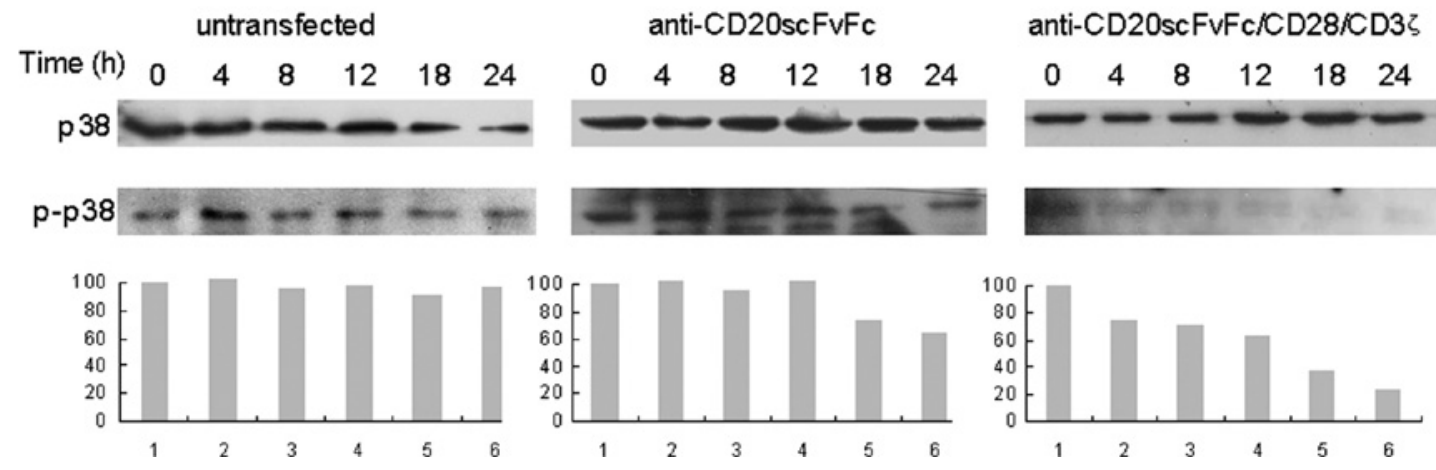

Figure 3. Treatment of Raji cells with engineered CD20-specific T cells down-regulated p-Lyn and p-p38 MAPK activity. (A) p-Lyn and Lyn protein expression in Raji cells at different time points. (B) p38 and p-p38 protein expression in Raji cells at different time points. The Western blots were analyzed by densitometry to show the relative levels of corresponding proteins.

against p38, p-p38, STAT3, p-STAT3, Lyn, p-Lyn, GAPDH and BCL-2 (Cell Signal Technology, MA, USA). Alternatively, whole cell lysates of engineered CD20-specific T cells were probed with a mouse anti-human $\mathrm{CD} 3 \zeta \mathrm{mAb}$ (Santa Cruz Biotechnology, Santa Cruz, CA, USA) (7).

Electrophoretic mobility shift assay for $N F-\kappa B$ and SP-1. Nuclear extracts from Raji cells by NE-PER Nuclear and Cytoplasmic Extraction Reagents (Thermo Fisher Scientific Inc., Rockford, IL, USA) were used to detect Sp1 and NF- $\kappa$ B. Electrophoretic mobility shift assay (EMSA) was performed using a LightShift Chemiluminescent EMSA kit (Thermo Fisher Scientific Inc., Rockford, IL, USA) according to the manufacturer's instructions. The following double-stranded oligonucleotides were used as the probe, SP1: 5'-ATT CGA TCG GGG CGG GGC GAG-3' and 3'-TAA GCT AGC CCC GCC CCG CTC-5'; NF-кB (2): 5'-AGT TGA GGG GAC TTT CCC AGG C-3' and 3'-TCA ACT CCC CTG AAA GGG TCC G-5'.

Statistical analysis. The data of each series of experiments were expressed as the mean \pm SD. Statistical differences between groups were analyzed using ANOVA analysis. $\mathrm{P}<0.05$ was considered to be statistically significant.

\section{Results}

Cytotoxicity of engineered CD20-specific T cells for targeting Raji cells. PTLs were transfected with vectors encoding antiCD20scFvFc or anti-CD20scFvFc/CD28/ל. The successful expression of the recombinant anti-CD20scFvFc/CD28/CD3 $\zeta$ protein was detected on anti-CD20scFvFc/CD28/CD3 $\zeta$ transfected PTLs by Western blot analysis of CD3 $\zeta$ expression (Fig. 1A). A $21-\mathrm{kDa}$ band corresponding to wildtype $\mathrm{CD} 3 \zeta$ was present in the cell lysates of anti-CD20scFvFc or anti-CD20scFvFc/CD28/CD3 $\}$-transfected PTLs. As shown in Fig. 1B, anti-CD20scFvFc/CD28/CD3\}-transfected PTLs lysed CD20-positive Raji cells with higher efficacy than antiCD20scFvFc-transfected PTLs, whereas untransfected PTLs had little effect on targeted cells.

Treatment of Raji cells with engineered CD20-specific $T$ cells inhibited IL-10 secretion. As shown in Fig. 2, IL-10 levels were up-regulated in a time-dependent manner in Raji cells following co-cultivation with untransfected $\mathrm{T}$ cells, whereas anti-CD20scFvFc or anti-CD20scFvFc/CD28/CD3 $\zeta$ transfected $\mathrm{T}$ cells led to the inhibition of IL-10 secretary in Raji cells. The treatment of Raji cells with anti-CD20scFvFc/ CD28/CD3-transfected T cells had a greater effect on the inhibition of IL-10 secretary than the treatment of Raji cells with anti-CD20scFvFc-transfected T cells.

Treatment of Raji cells with engineered CD20-specific T cells down-regulated p38 MAPK activity. Time kinetics analysis revealed that anti-CD20scFvFc/CD28/CD3 $\zeta$ transfected T cells inhibited p-Lyn as early as $8 \mathrm{~h}$ and was maximal at $24 \mathrm{~h}$ in Raji cells by Western blotting (Fig. 3A). Anti-CD20scFvFc-transfected $\mathrm{T}$ cells inhibited p-Lyn as early as $18 \mathrm{~h}$ and maximally at $24 \mathrm{~h}$ in Raji cells. Similarly, the treatment of Raji cells with anti-CD20scFvFc/CD28/ 
A
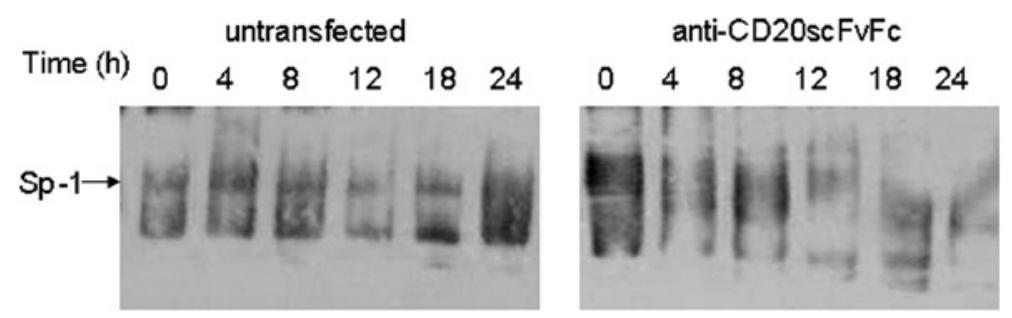

anti-CD20scFvFc/CD28/CD33

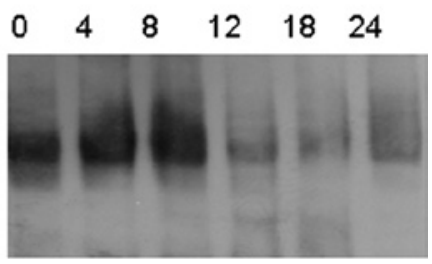

B
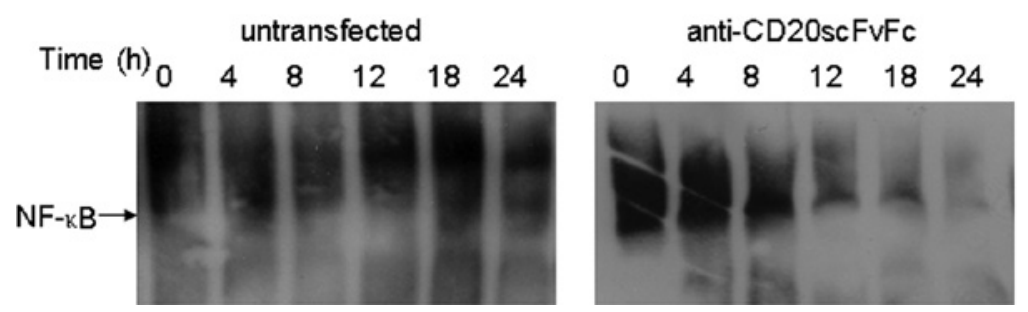

anti-CD20scFvFc/CD28/CD33

$\begin{array}{llllll}0 & 4 & 8 & 12 & 18 & 24\end{array}$

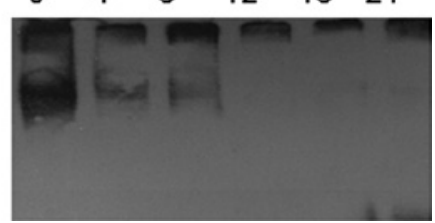

Figure 4. Treatment of Raji cells with engineered CD20-specific T cells inhibited Sp1 and NF-kB DNA-binding activities. (A) Sp1 DNA-binding and (B) NF-kB DNA-binding activities were determined in Raji cells at different time points.

A
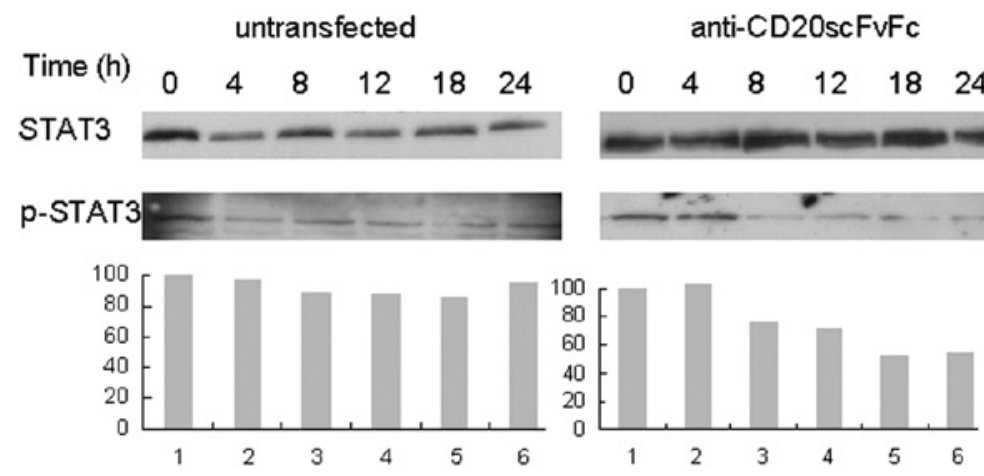

B
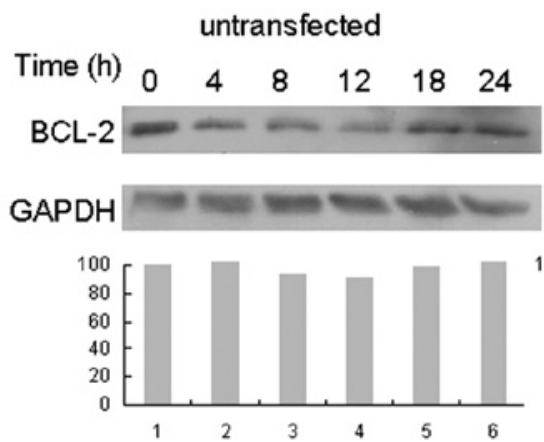

anti-CD20scFvFc
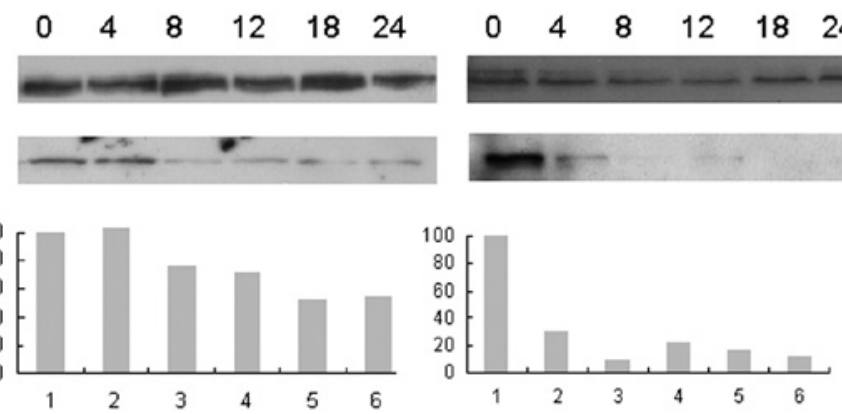

anti-CD20scFvFc/CD28/CD3?
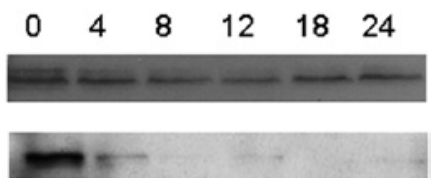

anti-CD20scFvFc/CD28/CD33

$\begin{array}{llllll}0 & 4 & 8 & 12 & 18 & 24\end{array}$
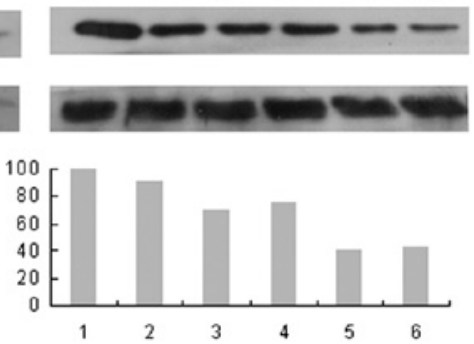

Figure 5. Treatment of Raji cells with engineered CD20-specific T cells down-regulated phosphor (p)-STAT3 and Bcl-2 expression levels. (A) p-STAT3 and STAT3 protein expression was determined in Raji cells at different time points. The Western blots were analyzed by densitometry to show the relative levels of corresponding proteins. (B) Bcl-2 and GAPDH protein expression was determined in Raji cells at different time points.

CD3 $\zeta$-transfected $\mathrm{T}$ cells markedly inhibited p-p38 as early as $4 \mathrm{~h}$, whereas the treatment of Raji cells with antiCD20scFvFc-transfected T cells slightly decreased p-p38 at 18 and $24 \mathrm{~h}$ (Fig. 3B). Treatment of Raji cells with modified or unmodified $\mathrm{T}$ cells showed no effect on the expression of Lyn and p38.

Treatment of Raji cells with engineered CD20-specific T cells decreased Spl and NF- $\kappa B$ DNA-binding activities. Gel shift assays revealed that the DNA-binding efficiency of Sp1 was significantly reduced in Raji cells treated with engineered CD20-specific T cells at $12 \mathrm{~h}$ (Fig. 4A). Additionally, the treatment of Raji cells with engineered CD20-specific T cells inhibited NF- $\kappa \mathrm{B}$ DNA-binding activity. Anti-CD20scFvFc/

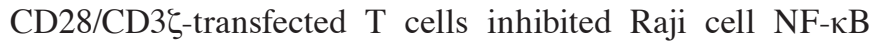
DNA-binding activity as early as $4 \mathrm{~h}$ and was maximal at $24 \mathrm{~h}$ (Fig. 4B). On the other hand, anti-CD20scFvFc-transfected $\mathrm{T}$ cells inhibited Raji cell NF- $\kappa \mathrm{B}$ DNA-binding activity as early as $12 \mathrm{~h}$. Treatment of Raji cells with anti-CD20scFvFc/ CD28/CD3 $\zeta$-transfected $\mathrm{T}$ cells showed a higher inhibition of 
Sp1 and NF-кB DNA-binding activities than treatment of Raji cells with anti-CD20scFvFc-transfected T cells.

Treatment of Raji cells with engineered CD20-specific $T$ cells inhibited phosphor-STAT3 and Bcl-2 expression. Fig. 5 shows that the treatment of Raji cells with antiCD20scFvFc- or anti-CD20scFvFc/CD28/CD3ל-transfected T cells inhibited p-STAT3 as early as 8 and $4 \mathrm{~h}$, respectively, and was maximal at $24 \mathrm{~h}$ (Fig. 5A). We observed that treatment of Raji cells with engineered CD20-specific $\mathrm{T}$ cells significantly inhibited Bcl-2 expression (Fig. 5B). Additionally, treatment of Raji cells with anti-CD20scFvFc/ CD28/CD3 3 -transfected T cells demonstrated a higher inhibition of p-STAT3 and Bcl-2 expression than treatment of Raji cells with anti-CD20scFvFc-transfected T cells.

\section{Discussion}

Adoptive T-cell immunotherapy of cancer is based on grafting cytotoxic $\mathrm{T}$ cells with chimeric antigen receptors constituting a tumor-specific single chain antibody ( $\mathrm{scFv}$ ) and a cellular activation intracellular signaling domain (10). Genetic modification of $\mathrm{T}$ cells with integral membrane scFv chimeric signaling receptors reacted with tumor-associated antigens in a non-MHC-restricted manner, thereby bypassing the $\mathrm{MHC}$ /peptide complex loss, which is a significant escape mechanism for most tumors (10-12). The intracellular signaling domain is derived from the cytoplasmic part of a membrane-bound receptor to induce cellular activation, e.g., CD3 $\zeta$ chain, which appears to have the most potency and to be sufficient for mediating T-cell activated signals (6). Tumor-specific cytotoxic T cells were successfully generated by the introduction of a chimeric $\mathrm{T}$-cell antigen receptor gene, consisting of an extracellular scFv and an intracellular part of a signaling molecule (CD3 $\zeta$ ) (13). Although engagement of $\zeta$ chains is sufficient to induce tumoricidal activity, without adequate co-stimulatory signals, it may not suffice to elicit substantial lymphocyte activation (3). Accumulated evidence has shown that $\mathrm{T}$ cells modified with chimeric antigen receptors incorporating a CD28 signaling domain are much more active when tested in vitro and in murine models $(6,10,12,14)$.

In the present study, a recombinant anti-CD20scFvFc/ CD28/CD3 $\zeta$ gene was constructed that provided both primary and costimulatory signals to $\mathrm{T}$ cells through the one chimera. This recombinant gene comprises the extracellular scFv linked to transmembrane and intracellular signaling domains of CD28 and CD3 $\zeta$ in tandem. Our group (7) has reported that engineered CD20-specific T cells, particularly lysed CD20positive target tumor cells, produced not only IFN- $\gamma$, but also IL-2 cytokines after binding to their target cells. Cytolysis of CD20-positive target cells by engineered CD20-specific $\mathrm{T}$ cells is antigen-specific since CD20-negative target cells (K562) were not lysed (7). Results of this study showed that the treatment of Raji cells with engineered CD20-specific T cells significantly inhibited IL-10 secretary. Treatment of Raji cells with anti-CD20scFvFc/CD28/CD3\}-transfected $T$ cells had a greater effect on the inhibition of IL-10 secretary than treatment of Raji cells with anti-CD20scFv-transfected $\mathrm{T}$ cells. Serum levels of IL-10 are elevated in a number of patients with NHL and a high IL-10 is associated with a poor rate of survival (15). Exogenous IL-10 significantly increases NHL tumor cell proliferation (16). As a protective factor, IL-10 enhances growth progression and aids in the pathogenesis of NHL through autocrine/paracrine loops (16-18).

The MAP kinase signaling pathway is involved in the regulation of IL-10 production in Burkitt lymphoma cell lines $(19,20)$. Elevated levels of activated MAP kinase have been observed in a variety of solid tumors and hematologic malignancies, and MAP kinases may be important in the development of new therapeutic approaches $(2,20)$. The antiCD20 monoclonal antibody rituximab triggers and modifies various intracellular signaling pathways in NHL B-cell lines, resulting in the induction of apoptosis and chemosensitization $(2,21,22)$. Alas et al (18) reported that rituximab downregulated tumor-derived IL-10 transcription and subsequently down-regulated Bcl-2 gene expression. Previous findings suggest that IL-10 plays a role in the expression of the antiapoptotic Bcl-2 gene product via STAT3 activation $(16,23)$. Rituximab induces the inhibition of IL-10 transcription and secretion. Rituximab also induces the inhibition of STAT3 activity and Bcl-2 expression, as well as sensitization to the apoptotic effects of various chemotherapeutic drugs, through the p38 MAPK pathway (2). Thus, our study examined p38 MAPK signaling pathways mediated by genetically modified CD20-specific T cells in the Raji cell line. Engineered CD20-specific T cells inhibited p-Lyn and p38 MAPK activities, and decreased Sp1 and IL-10 levels in targeted Raji-cells. In addition, genetically-modified $\mathrm{T}$ cells reduced $\mathrm{NF}-\kappa \mathrm{B}$ DNA-binding activities and down-regulated p-STAT3 and Bcl-2 expression levels. These data demonstrated the role of p38 MAPK and $\mathrm{NF}-\kappa \mathrm{B}$ signaling pathways in the regulation of IL-10 transcription.

Vega et al (2) reported that the down-regulation of $\mathrm{NF}-\kappa \mathrm{B}$ activity induced by rituximab was mediated through the p38 MAPK signaling pathway, and that phosphor-Lyn and p38 MAPK activities were inhibited by rituximab, resulting in the inhibition of IL-10 transcription via Sp1 (2). Consequently, down-regulation of the autocrine/paracrine loop of IL-10/IL-10R signaling partially inhibited p-STAT3 and Bcl-2 expression (2). Sp1 transcription factor is activated by $\mathrm{p} 38$ MAPK and Sp1 is involved in the regulation of IL-10 expression in a number of cell lines (24). Moreover, our study showed that the treatment of Raji cells with engineered T cells expressing anti-CD20scFvFc/CD28/CD3 $\zeta$ led to a stronger inhibition of p38 MAPK activity and down-regulation of Bcl-2 expression and IL-10 secretary than the treatment of Raji cells with engineered $\mathrm{T}$ cells expressing anti-CD20scFvFc. These results demonstrate that $\mathrm{CD} 3 \zeta$ and $\mathrm{CD} 28$ co-stimulation signaling fusion with anti-CD20 immunoreceptor in grafted $\mathrm{T}$ cells synergistically enhances target cytotoxicity, inhibits p38 MAPK activity and decreases IL-10 secretary in the target tumor cells.

In conclusion, our results showed that treatment of Raji cells with engineered CD20-specific $\mathrm{T}$ cells inhibited p38 MAPK and NF- $\kappa$ B DNA-binding activities, resulting in the inhibition of IL-10 secretion, p-STAT3 and Bcl-2 expression. These results indicated that modifications of the cellular p38 MAPK signaling pathways in target cells by engineered CD20-specific T cells may be crucial for the antitumor effect 
of adoptive T-cell therapy. Findings of the abovementioned studies showed that combining chimeric CD3 $\zeta$ and CD28 with an anti-CD20 immunoreceptor in grafted T cells led to improved activity in vitro, compared to anti-CD20 immunoreceptor alone in grafted $\mathrm{T}$ cells. This process is therefore expected to substantially enhance the antitumor efficacy of genetically-modified T cells. However, it should be noted that this study has examined only the non-Hodgkin's lymphoma Raji cell line, in vitro. Thus, further investigation of the activity of these modified T cells in vivo is required.

\section{Acknowledgements}

The authors would like to thank Drs Daming Shan and Hinrich Abken for kindly donating the pLNCX and pBULLET vectors.

\section{References}

1. Vega MI, Huerta-Yepez S, Jazirehi AR, Garban H and Bonavida B: Rituximab (chimeric anti-CD20) sensitizes B-NHL cell lines to Fas-induced apoptosis. Oncogene 24: 8114-8127, 2005.

2. Vega MI, Huerta-Yepaz S, Garban H, Jazirehi A, Emmanouilides $\mathrm{C}$ and Bonavida B: Rituximab inhibits p38 MAPK activity in 2F7 B NHL and decreases IL-10 transcription: pivotal role of p38 MAPK in drug resistance. Oncogene 23: 3530-3540, 2004.

3. Till BG and Press OW: Treatment of lymphoma with adoptively transferred T cells. Expert Opin Biol Ther 9: 1407-1425, 2009.

4. Jensen MC, Cooper LJ, Wu AM, Forman SJ and Raubitschek A Engineered CD20-specific primary human cytotoxic T lymphocytes for targeting B-cell malignancy. Cytotherapy 5: 131-138, 2003.

5. Eshhar Z, Waks T, Gross G and Schindler DG: Specific activation and targeting of cytotoxic lymphocytes through chimeric single chains consisting of antibody-binding domains and the gamma or zeta subunits of the immunoglobulin and $\mathrm{T}$ cell receptors. Proc Natl Acad Sci USA 90: 720-724, 1993.

6. Hombach A, Wieczarkowiecz A, Marquardt T, et al: Tumorspecific $\mathrm{T}$ cell activation by recombinant immunoreceptors: CD3 zeta signaling and CD28 costimulation are simultaneously required for efficient IL-2 secretion and can be integrated into one combined CD28/CD3 zeta signaling receptor molecule. J Immunol 167: 6123-6131, 2001.

7. Yu K, Hu Y, Tan Y, et al: Immunotherapy of lymphomas with $\mathrm{T}$ cells modified by anti-CD20 scFv/CD28/CD3zeta recombinant gene. Leuk Lymphoma 49: 1368-1373, 2008.

8. Zheng Y, Yu K, Du J, et al: Potential therapeutic strategy for non-Hodgkin lymphoma by anti-CD20scFvFc/CD28/CD3zeta gene transfected T cells. J Exp Clin Cancer Res 29: 121, 2010.

9. Jiang L, Chen Y, Chan CY, et al: Down-regulation of stathmin is required for TGF-beta inducible early gene 1 induced growth inhibition of pancreatic cancer cells. Cancer Lett 274: 101-108, 2009.
10. Wang $\mathrm{H}$, Wei $\mathrm{H}$, Zhang $\mathrm{R}$, et al: Genetically targeted $\mathrm{T}$ cells eradicate established breast cancer in syngeneic mice. Clin Cancer Res 15: 943-950, 2009.

11. Teng MW, Kershaw MH, Moeller M, Smyth MJ and Darcy PK: Immunotherapy of cancer using systemically delivered genemodified human T lymphocytes. Hum Gene Ther 15: 699-708, 2004.

12. Haynes NM, Trapani JA, Teng MW, et al: Single-chain antigen recognition receptors that costimulate potent rejection of established experimental tumors. Blood 100: 3155-3163, 2002.

13. Rossig $\mathrm{C}$ and Brenner MK: Genetic modification of T lymphocytes for adoptive immunotherapy. Mol Ther 10: 5-18, 2004.

14. Finney HM, Akbar AN and Lawson AD: Activation of resting human primary $\mathrm{T}$ cells with chimeric receptors: costimulation from CD28, inducible costimulator, CD134, and CD137 in series with signals from the TCR zeta chain. J Immunol 172: 104-113, 2004.

15. El-Far M, Fouda M, Yahya R and el-Baz H: Serum IL-10 and IL-6 levels at diagnosis as independent predictors of outcome in non-Hodgkin's lymphoma. J Physiol Biochem 60: 253-258, 2004.

16. Voorzanger N, Touitou R, Garcia E, et al: Interleukin (IL)-10 and IL-6 are produced in vivo by non-Hodgkin's lymphoma cells and act as cooperative growth factors. Cancer Res 56: 5499-5505, 1996.

17. Benjamin D, Park CD and Sharma V: Human B cell interleukin 10. Leuk Lymphoma 12: 205-210, 1994.

18. Alas S, Emmanouilides $C$ and Bonavida B: Inhibition of interleukin 10 by rituximab results in down-regulation of bcl-2 and sensitization of B-cell non-Hodgkin's lymphoma to apoptosis. Clin Cancer Res 7: 709-723, 2001.

19. Vockerodt M, Haier B, Buttgereit P, Tesch H and Kube D: The Epstein-Barr virus latent membrane protein 1 induces interleukin-10 in Burkitt's lymphoma cells but not in Hodgkin's cells involving the p38/SAPK2 pathway. Virology 280: 183-198, 2001.

20. Platanias LC: Map kinase signaling pathways and hematologic malignancies. Blood 101: 4667-4679, 2003.

21. Pedersen IM, Buhl AM, Klausen P, Geisler CH and Jurlander $\mathrm{J}$ : The chimeric anti-CD20 antibody rituximab induces apoptosis in B-cell chronic lymphocytic leukemia cells through a p38 mitogen activated protein-kinase-dependent mechanism. Blood 99: 1314-1319, 2002.

22. Jazirehi AR, Gan XH, De Vos S, Emmanouilides $\mathrm{C}$ and Bonavida B: Rituximab (anti-CD20) selectively modifies Bcl-xL and apoptosis protease activating factor-1 (Apaf-1) expression and sensitizes human non-Hodgkin's lymphoma $\mathrm{B}$ cell lines to paclitaxel-induced apoptosis. Mol Cancer Ther 2: 1183-1193, 2003.

23. Alas S and Bonavida B: Rituximab inactivates signal transducer and activation of transcription 3 (STAT3) activity in B-nonHodgkin's lymphoma through inhibition of the interleukin 10 autocrine/paracrine loop and results in down-regulation of Bcl-2 and sensitization to cytotoxic drugs. Cancer Res 61: 5137-5144, 2001.

24. Ma W, Lim W, Gee K, et al: The p38 mitogen-activated kinase pathway regulates the human interleukin-10 promoter via the activation of Sp1 transcription factor in lipopolysaccharidestimulated human macrophages. J Biol Chem 276: 13664-13674, 2001. 